\title{
On the geometry of Hele-Shaw flows with small surface tension
}

\author{
AleXANDER VASIL’EV ${ }^{\dagger}$ AND IRINA MarkinA \\ Departamento de Matemática, Universidad Técnica Federico Santa María, \\ Casilla 110-V, Valparaíso, Chile
}

[Received 28 March 2001 and in revised form 20 January 2003]

\begin{abstract}
We study the time evolution of the free boundary of a viscous fluid in the non-zero-surface-tension models for planar flows in Hele-Shaw cells under injection. Applying methods of conformal map we prove that certain geometric properties, such as starlikeness and directional convexity, are preserved in time.
\end{abstract}

Keywords: Free boundary problem; Hele-Shaw flow; surface tension; conformal map.

2000 Mathematics Subject Classification: Primary 76S05, 76D27, 30C45; Secondary 35Q35, 30C35.

\section{Introduction}

We discuss the Hele-Shaw problem in the plane in two basic cases. The first one deals with the classical situation of injection through a unique source in a finite region. The second one is concerned with the free boundary extending to the point at infinity. Starting with the earlier works of L. A. Galin [9] and P. Ya. Polubarinova-Kochina [19, 20], various aspects of the planar Hele-Shaw viscous flows with vanishing surface tension were investigated by a number of scientists. It is known [31] that in the zero-surface-tension Hele-Shaw problem with an initial region with an analytic boundary the classical solution exists locally in time. Recently [25], it became clear that the model could be interpreted as a particular case of the abstract Cauchy problem, and thus, the classical solvability (locally in time) may be proved using the nonlinear abstract Cauchy-Kovalevskaya Theorem.

One asks whether a non-zero-surface-tension model approximates the zero-surface-tension one. The answer is negative in the case of a receding fluid (see numerical evidence in [1, 24]). In the case of injection the answer is supposed to be affirmative but is still unknown. In our paper we consider geometric properties that are preserved under injection during the time evolution of zero- as well as of non-zero-surface-tension models.

For the non-zero-surface-tension case J. Duchon and R. Robert [3] proved the local existence in time of the weak solution for bounded domains. Recently, G. Prokert [23] obtained even global existence in time and exponential decay of the solution near equilibrium (when surface tension is a unique driving mechanism). The results are obtained in Sobolev spaces $H^{s}$ with sufficiently large $s$. J. Escher and G. Simonett [5, 6] proved the local existence, uniqueness and regularity of classical solutions to one- and two-phase Hele-Shaw problems with surface tension when the initial domain has a smooth boundary. The global existence in the case of the phase domain close to a disk was

\footnotetext{
${ }^{\dagger}$ E-mail: alexander.vasiliev@mat.utfsm.cl

${ }^{\ddagger}$ E-mail: irina.markina@mat.utfsm.cl

This work is partially supported by Projects Fondecyt (Chile) \# 1020067 and 1030373.
} 
proved in [7]. For unbounded domains with unbounded boundary the corresponding result about short-time existence and uniqueness for positive surface tension has been obtained by M. Kimura [15]. More about the local existence of classical solutions can be found in [30].

In the present paper we derive equations for the free boundaries either for unbounded domains (similarly to [8]) whose free boundary extends to infinity, or for the classical situation of suction and injection, in non-zero-surface-tension models. Using methods of geometric function theory we prove that some geometric properties of the free boundary that depend on those of the initial one are preserved as long as the classical solution exists.

\section{Bounded case}

A simple dimensionless model of a moving viscous incompressible fluid in a plane Hele-Shaw cell is described by a potential flow with velocity field $V=\left(V_{1}, V_{2}\right)$. We assume that a unique force acting in this field is the dimensionless pressure $p$ which is the potential for the fluid velocity

$$
V=-\frac{h^{2}}{12 \mu} \nabla p,
$$

where $h$ is the cell gap and $\mu$ is the viscosity of the fluid (see e.g. [18, 27]). Denote by $\Omega(t)$ a bounded simply connected domain in $\mathbb{C}$ occupied by the moving fluid and by $\Gamma(t)$ its moving boundary. Suction or injection is carried out through a point sink or source placed at the origin $0 \in \Omega(t)$. The initial boundary $\Gamma_{0} \equiv \Gamma(0)$ is supposed to be analytic and smooth. We have a homogeneous sink/source with the normalization $p(z, t) \sim \frac{Q}{2 \pi} \log |z|$ about the origin. The incompressibility of the fluid implies the harmonicity of the pressure $p(z, t)$ except for a singular point at the origin,

$$
\Delta p=-Q \delta_{0}(z), \quad z=x+i y \in \Omega(t),
$$

where $\delta_{0}(z)$ stands for the standard Dirac distribution with singularity at the origin. The value $Q$ is the strength of the sink (source), $Q>0$ in the case of a sink (and $Q<0$ in the case of a source). The non-zero-surface-tension dynamic boundary condition is given by the product of the surface tension $\gamma$ and the mean curvature $\kappa$ in the $z$-plane (J. W. McLean and P. G. Saffman [16]),

$$
p(z)=\gamma \kappa \quad \text { for } z \in \Gamma(t) .
$$

In a real Hele-Shaw cell, (2) is an approximation to the effect of complicated three-dimensional free surface flow near the moving interface. The normal velocity on the boundary is given by the kinematic condition for the normal velocity

$$
v_{n}=-\frac{\partial p}{\partial \vec{n}}
$$

where $\vec{n}$ is the outward unit normal vector to $\Gamma(t)$. The problem (1)- 3 has a unique classical solution locally in time for both positive and negative $Q$ [6, 30].

The zero-surface-tension problem $(\gamma=0)$ with suction is ill-posed in the Hadamard sense. This means that an arbitrarily small perturbation of the initial domain $\Omega_{0} \equiv \Omega(0)$ can produce an $O(1)$ effect in arbitrarily short time. The injection problem is well-posed (C. M. Elliott and V. Janovský [4]). The condition (2) is one of the proposals for the regularization of the ill-posed problem (which 
is also known as the Laplace-Young condition). It accounts for the influence of surface tension on the pressure across the interface.

We consider the complex potential $W=W(x, y, t) \equiv W(z, t)$, Re $W=p$. For each fixed $t$ it is an analytic function defined in $\Omega(t)$ whose real part solves the problem (1)-(2). In the neighbourhood of the origin we have the expansion

$$
W(z, t)=\frac{Q}{2 \pi} \log z+w(z, t),
$$

where $w(z, t)$ is an analytic regular function in $\Omega(t)$. We have

$$
\frac{\partial W}{\partial z}=\frac{\partial p}{\partial x}-i \frac{\partial p}{\partial y},
$$

by the Cauchy-Riemann conditions.

In order to derive the equation for the free boundary $\Gamma(t)$ we consider an auxiliary parametric complex $\zeta$-plane, $\zeta=\xi+i \eta$. The Riemann Mapping Theorem shows that there exists a unique conformal univalent map $f(\zeta, t)$ of the unit disk $U=\{\zeta:|z|<1\}$ onto the phase domain, $f: U \rightarrow \Omega(t)$, with $f(0, t) \equiv 0, f^{\prime}(0, t)>0$. The function $f(\zeta, t)$ gives the parameterization $\Gamma(t)=\left\{f\left(e^{i \theta}, t\right): \theta \in[0,2 \pi)\right\}$ of the moving boundary.

The outward unit normal vector in (3) is given as

$$
\vec{n}=\zeta \frac{f^{\prime}(\zeta, t)}{\left|f^{\prime}(\zeta, t)\right|}, \quad \zeta \in \partial U
$$

From now on, we use the notations $\dot{f}=\partial f / \partial t, f^{\prime}=\partial f / \partial \zeta$. Thus, 3 , and 5 lead to the formula

$$
v_{n}=V \cdot \vec{n}=-\operatorname{Re}\left(\frac{\partial W}{\partial z} \zeta \frac{f^{\prime}(\zeta, t)}{\left|f^{\prime}(\zeta, t)\right|}\right)
$$

The superposition $W \circ f(\zeta, t)$ is an analytic function in the unit disk. Since the Laplacian is invariant under conformal map, the solution to (1)-(2) is given in terms of the $\zeta$-plane as

$$
W \circ f(\zeta, t)=\frac{Q}{2 \pi} \log \zeta+\frac{\gamma}{2 \pi} \int_{0}^{2 \pi} \kappa\left(\mathrm{e}^{i \theta}, t\right) \frac{\mathrm{e}^{i \theta}+\zeta}{\mathrm{e}^{i \theta}-\zeta} \mathrm{d} \theta+i C,
$$

where

We calculate

$$
\kappa\left(\mathrm{e}^{i \theta}, t\right)=\frac{\operatorname{Re}\left(1+\mathrm{e}^{i \theta} f^{\prime \prime}\left(\mathrm{e}^{i \theta}, t\right) / f^{\prime}\left(\mathrm{e}^{i \theta}, t\right)\right)}{\left|f^{\prime}\left(\mathrm{e}^{i \theta}, t\right)\right|}, \quad \theta \in[0,2 \pi) .
$$

$$
\frac{\partial \kappa\left(\mathrm{e}^{i \theta}, t\right)}{\partial \theta}=\frac{-\operatorname{Im} \mathrm{e}^{2 i \theta} S_{f}\left(\mathrm{e}^{i \theta}, t\right)}{\left|f^{\prime}\left(\mathrm{e}^{i \theta}, t\right)\right|},
$$

where $S_{f}$ is the Schwarzian derivative. Differentiating (6) we deduce that

$$
\zeta \frac{\partial W}{\partial z} f^{\prime}(\zeta, t)=\frac{Q}{2 \pi}+\frac{\gamma}{\pi} \int_{0}^{2 \pi} \frac{\kappa\left(\mathrm{e}^{i \theta}\right) \zeta \mathrm{e}^{i \theta}}{\left(\mathrm{e}^{i \theta}-\zeta\right)^{2}} \mathrm{~d} \theta, \quad \zeta \in U .
$$

Integrating by parts we obtain

$$
\zeta \frac{\partial W}{\partial z} f^{\prime}(\zeta, t)=\frac{Q}{2 \pi}-\frac{\gamma}{2 \pi i} \int_{0}^{2 \pi} \frac{\operatorname{Im} \mathrm{e}^{2 i \theta} S_{f}\left(\mathrm{e}^{i \theta}, t\right)}{\left|f^{\prime}\left(\mathrm{e}^{i \theta}, t\right)\right|} \frac{\mathrm{e}^{i \theta}+\zeta}{\mathrm{e}^{i \theta}-\zeta} \mathrm{d} \theta .
$$


Then we apply the Sokhotskiı̌-Plemelj formulae [17] and, finally, deduce that

$$
\begin{aligned}
\operatorname{Re} \dot{f}(\zeta, t) \overline{\zeta f^{\prime}(\zeta, t)} & =-\operatorname{Re}\left(\frac{\partial W}{\partial z} \circ f(\zeta, t)\right) \zeta f^{\prime}(\zeta, t) \\
& =-\frac{Q}{2 \pi}-\gamma H\left[i \operatorname{Im} \frac{\zeta^{2} S_{f}(\zeta, t)}{\left|f^{\prime}(\zeta, t)\right|}\right](\theta), \quad \zeta=\mathrm{e}^{i \theta}
\end{aligned}
$$

where the Hilbert transform in (7) is of the form

$$
H[\psi](\theta):=\frac{1}{\pi} \mathrm{P} \cdot \mathrm{V} \cdot \theta \int_{0}^{2 \pi} \frac{\psi\left(\mathrm{e}^{i \theta^{\prime}}\right)}{1-\mathrm{e}^{i\left(\theta-\theta^{\prime}\right)}} \mathrm{d} \theta^{\prime} .
$$

This type of equation is known as the Polubarinova-Galin equation (see e.g. [13]). In terms of the equation (7) the existence of a classical solution means that the parametric function $f(\zeta, t)$ is analytic in a neighbourhood of the disk $U$ and univalent in $U$ locally in time (see e.g. [24, 25]).

\section{Geometric properties of the free boundary}

Several authors studied starlike dynamics under injection. For example, some authors imposed "strict starlikeness" as an additional condition to prove short-time existence and uniqueness of a weak or classical solution (see, e.g., [23, 26]). Let us refer also to the paper by E. Di Benedetto and A. Friedmann [2] where the authors discussed the weak solution and proved that starting with a domain $\Omega_{0}$ that is starlike with respect to a small ball centred at the point of the source the evolutionary domains $\Omega(t)$ remain starlike with respect to a ball (maybe of different radius) in the zero-surface-tension case. If the classical solution exists, then it is the same as the weak one (see, e.g., [11]). Therefore, the same is true for the classical solution. In [12] the same result is proved but for domains which are starlike with respect to a point source, using complex variable methods. Here we generalize this result to non-zero surface tension using a slightly different method.

We recall that a domain $\Omega \subset \mathbb{C}$ with $0 \in \Omega$ is said to be starlike (with respect to the origin) if each ray starting at the origin intersects $\Omega$ in a set that is either a line segment or a ray. A function $f$ mapping $U$ onto a starlike domain is said to be starlike $\left(f \in S^{*}\right)$, and it satisfies the necessary and sufficient condition of starlikeness (see [10,21, 22])

$$
\operatorname{Re} \frac{\zeta f^{\prime}(\zeta)}{f(\zeta)}>0, \quad \zeta \in U
$$

If the function $f$ can be extended analytically onto the closure $\bar{U}$ of $U$, then the inequality sign in (8) can be replaced by $\geqslant$ where the equality can be attained at the unit circle $\partial U$.

We need a technical formula. For the function $f: U \rightarrow \mathbb{C}$ which parameterizes the phase domain $\Omega(t)$ we have the equality

$$
\frac{\partial}{\partial \theta} H\left[\frac{i \mathrm{e}^{2 i \theta} \operatorname{Im} S_{f}\left(\mathrm{e}^{i \theta}\right)}{\left|f^{\prime}\left(\mathrm{e}^{i \theta}\right)\right|}\right](\theta)=-H[i A](\theta)
$$

with

$$
A(\zeta)=\frac{\operatorname{Re}\left(2 \zeta^{2} S_{f}(\zeta)+\zeta\left[\left(\frac{f^{\prime \prime}(\zeta)}{f^{\prime}(\zeta)}\right)^{\prime \prime}-\frac{f^{\prime \prime}(\zeta)}{f^{\prime}(\zeta)}\left(\frac{f^{\prime \prime}(\zeta)}{f^{\prime}(\zeta)}\right)^{\prime}\right]\right)+\operatorname{Im} \frac{\zeta f^{\prime \prime}(\zeta)}{f^{\prime}(\zeta)} \operatorname{Im} \zeta^{2} S_{f}(\zeta)}{\left|f^{\prime}(\zeta)\right|}
$$

We denote by $T$ the blow-up time (the classical solution exists during the period $[0, T)$ ). 
THEOREM 1 Let $Q<0$ and let the surface tension $\gamma$ be sufficiently small. If the initial domain $\Omega_{0}$ is starlike, then there exists $t=t(\gamma) \leqslant T$ such that for each $t \in[0, t(\gamma)]$ the domain $\Omega(t)$ is also starlike.

Proof. Suppose that there exists a critical map $f \in S^{*}$. Saying this we mean that the image of $U$ under the map $\zeta f^{\prime}(\zeta, t) / f(\zeta, t),|\zeta| \leqslant 1$, touches the imaginary axis and there exist $t^{\prime} \geqslant 0$ and $\zeta_{0}=\mathrm{e}^{i \theta_{0}}$ such that

$$
\arg \frac{\zeta_{0} f^{\prime}\left(\zeta_{0}, t^{\prime}\right)}{f\left(\zeta_{0}, t^{\prime}\right)}=\frac{\pi}{2} \quad\left(\text { or }=-\frac{\pi}{2}\right),
$$

and for any $\varepsilon>0$ there are $t>t^{\prime}$ and $\theta \in\left(\theta_{0}-\varepsilon, \theta_{0}+\varepsilon\right)$ such that

$$
\arg \frac{\mathrm{e}^{i \theta} f^{\prime}\left(\mathrm{e}^{i \theta}, t\right)}{f\left(\mathrm{e}^{i \theta}, t\right)} \geqslant \frac{\pi}{2} \quad\left(\text { or } \leqslant-\frac{\pi}{2}\right) .
$$

For definiteness we put the sign $(+)$ in 10 . Without loss of generality, assume $t^{\prime}=0$. Since $f^{\prime}\left(\mathrm{e}^{i \theta}, t\right) \neq 0$, our assumption about the sign in 10 yields

$$
\operatorname{Im} \frac{\zeta_{0} f^{\prime}\left(\zeta_{0}, 0\right)}{f\left(\zeta_{0}, 0\right)}>0
$$

(the negative case is considered similarly).

Since $\zeta_{0}$ is a critical point and the image of the unit disk $U$ under the mapping $\zeta f^{\prime}(\zeta, 0) / f(\zeta, 0)$ touches the imaginary axis at the point $\zeta_{0}=\mathrm{e}^{i \theta_{0}}$, we deduce that

$$
\left.\frac{\partial}{\partial \theta} \arg \frac{\mathrm{e}^{i \theta} f^{\prime}\left(\mathrm{e}^{i \theta}, 0\right)}{f\left(\mathrm{e}^{i \theta}, 0\right)}\right|_{\theta=\theta_{0}}=0,\left.\quad \frac{\partial}{\partial r} \arg \frac{r \mathrm{e}^{i \theta_{0}} f^{\prime}\left(r \mathrm{e}^{i \theta_{0}}, 0\right)}{f\left(r \mathrm{e}^{i \theta}, 0\right)}\right|_{r=1} \geqslant 0 .
$$

We calculate

$$
\begin{aligned}
& \operatorname{Re}\left[1+\frac{\zeta_{0} f^{\prime \prime}\left(\zeta_{0}, 0\right)}{f^{\prime}\left(\zeta_{0}, 0\right)}-\frac{\zeta_{0} f^{\prime}\left(\zeta_{0}, 0\right)}{f\left(\zeta_{0}, 0\right)}\right]=0 \\
& \operatorname{Im}\left[1+\frac{\zeta_{0} f^{\prime \prime}\left(\zeta_{0}, 0\right)}{f^{\prime}\left(\zeta_{0}, 0\right)}-\frac{\zeta_{0} f^{\prime}\left(\zeta_{0}, 0\right)}{f\left(\zeta_{0}, 0\right)}\right] \geqslant 0
\end{aligned}
$$

One can derive

$$
\frac{\partial}{\partial t} \arg \frac{\zeta f^{\prime}(\zeta, t)}{f(\zeta, t)}=\operatorname{Im} \frac{\partial}{\partial t} \log \frac{f^{\prime}(\zeta, t)}{f(\zeta, t)}=\operatorname{Im}\left(\frac{\frac{\partial}{\partial t} f^{\prime}(\zeta, t)}{f^{\prime}(\zeta, t)}-\frac{\frac{\partial}{\partial t} f(\zeta, t)}{f(\zeta, t)}\right) .
$$

Now we differentiate the equation (7) with respect to $\theta$ using (9):

$$
\operatorname{Im}\left(\overline{f^{\prime}(\zeta, t)} \frac{\partial}{\partial t} f^{\prime}(\zeta, t)-\overline{\zeta f^{\prime}(\zeta, t)} \dot{f}(\zeta, t)-\overline{\zeta^{2} f^{\prime \prime}(\zeta, t)} \dot{f}(\zeta, t)\right)=-\gamma H[i A](\theta)
$$

for $\zeta=\mathrm{e}^{i \theta}$. This equality is equivalent to the following:

$$
\begin{aligned}
\left|f^{\prime}(\zeta, t)\right|^{2} \operatorname{Im}\left(\frac{\frac{\partial}{\partial t} f^{\prime}(\zeta, t)}{f^{\prime}(\zeta, t)}-\frac{\frac{\partial}{\partial t} f(\zeta, t)}{f(\zeta, t)}\right)= & \operatorname{Im}\left(\overline{\zeta f^{\prime}(\zeta, t)} \dot{f}(\zeta, t)\right)\left(\frac{\overline{\zeta f^{\prime \prime}(\zeta, t)}}{f^{\prime}(\zeta, t)}-\frac{\zeta f^{\prime}(\zeta, t)}{f(\zeta, t)}+1\right) \\
& -\gamma H[i A](\theta) .
\end{aligned}
$$


Substituting (7) and $(13)$ in the latter expression, we finally have

$$
\begin{aligned}
\left.\frac{\partial}{\partial t} \arg \frac{\zeta f^{\prime}(\zeta, t)}{f(\zeta, t)}\right|_{\zeta=\mathrm{e}^{i \theta_{0},}, t=0} & =\frac{1}{\left|f^{\prime}\left(\mathrm{e}^{i \theta_{0}}, 0\right)\right|^{2}}\left(\frac{Q}{2 \pi}+\gamma H\left[i \operatorname{Im} \frac{\mathrm{e}^{2 i \theta} S_{f}\left(\mathrm{e}^{i \theta}, t\right)}{\left|f^{\prime}\left(\mathrm{e}^{i \theta}, t\right)\right|}\right]\left(\theta_{0}\right)\right) \\
\times & \operatorname{Im}\left(\frac{\mathrm{e}^{i \theta_{0}} f^{\prime}\left(\mathrm{e}^{i \theta_{0}}, 0\right)}{f\left(\mathrm{e}^{i \theta_{0}}, 0\right)}+\frac{\mathrm{e}^{i \theta_{0}} f^{\prime \prime}\left(\mathrm{e}^{i \theta_{0}}, 0\right)}{f^{\prime}\left(\mathrm{e}^{i \theta_{0}}, 0\right)}\right)-\gamma \frac{H\left[i A\left(\mathrm{e}^{i \theta}\right)\right]\left(\theta_{0}\right)}{\left|f^{\prime}\left(\mathrm{e}^{i \theta_{0}}, 0\right)\right|^{2}} .
\end{aligned}
$$

The right-hand side of this equality is strictly negative for small $\gamma$ because of (12), (14). Since we consider the set of critical points in the compact set $\partial U$, we can choose $\gamma_{0}>0$ to be minimal such that the right-hand side is negative for all critical points and $\gamma \in\left[0, \gamma_{0}\right]$. Therefore,

$$
\arg \frac{\mathrm{e}^{i \theta_{0}} f^{\prime}\left(\mathrm{e}^{i \theta_{0}}, t\right)}{f\left(\mathrm{e}^{i \theta_{0}}, t\right)}<\frac{\pi}{2}
$$

for $t>0$ (close to 0 ). By continuity, the same inequality holds in some neighbourhood of any critical point $\theta_{0}$. For all $\theta$ outside the above mentioned neighbourhoods the same inequality is valid because

$$
\arg \frac{\mathrm{e}^{i \theta} f^{\prime}\left(\mathrm{e}^{i \theta}, t\right)}{f\left(\mathrm{e}^{i \theta}, t\right)}-\frac{\pi}{2} \leqslant-\delta<0 .
$$

This contradicts the assumption of the existence of critical points and ends the proof.

\section{Discussion}

1) An interesting question appears when $\gamma \rightarrow 0$. It turns out that the solution in the limiting $\gamma$-surface-tension case need not always be the corresponding zero-surface-tension solution at least in the problem of receding fluid (see the discussion in [28, 29, 30]). This means that if $\Omega(t, \gamma)$ stands for the moving fluid domain at time $t$ with surface tension $\gamma$, then assuming $\lim _{\gamma \rightarrow 0} \Omega(t, \gamma)=\Omega(t, 0)$, the domain $\Omega(0,0)$ is not necessarily the limit of $\Omega(0, \gamma)$ as $\gamma \rightarrow 0$. Theorem 1 shows that some geometry remains the same as $\gamma \rightarrow 0$. In particular, in the zero-surface-tension model $t(0)=T$ and starlikeness is preserved for the whole time $t \in[0, T)$.

2) The result of Theorem 1 is local in the sense that the starlikeness in question remains the same in some neighbourhood of the initial instant when the domain $\Omega_{0}$ is starlike. This neighbourhood depends either on $\gamma$ or else on the properties of the boundary $\Gamma_{0}$ involving the value of $H\left[i \zeta^{2} \operatorname{Im} S_{f} /\left|f^{\prime}\right|\right](\theta)$ and $H[i A](\theta)$. We also point out that in the case of the zero-surface-tension model, $Q<0$ corresponds to the situation when the travelling wave solution is linearly stable [13] for $t \in[0, T)$.

3) There are expectations that Theorem 1 is still true for large $\gamma$. Unfortunately, we are not able to prove this at the moment. The influence of the curvature makes it less probable that $t(\gamma)=t_{0}$ for $\gamma \neq 0$ but $t(\gamma)$ could exist even for large $\gamma$. The restriction on the smallness of $\gamma$ comes from the method of proof.

\section{Isoperimetric inequalities}

Let $S(t)$ be the area of the domain $\Omega(t)$ in the Hele-Shaw dynamics under the conditions of Section 2. A simple application of the Green theorem implies that the rate of the area change is 
$\dot{S}=-Q$. Let $\gamma=0, Q<0$. From 77 we deduce that

$$
\dot{a}=-a \frac{1}{4 \pi^{2}} \int_{0}^{2 \pi} \frac{Q}{\left|f^{\prime}\left(\mathrm{e}^{i \theta}, t\right)\right|^{2}} \mathrm{~d} \theta \geqslant-a \frac{1}{4 \pi^{2}} \int_{0}^{2 \pi} \operatorname{Re} \frac{Q}{f^{\prime}\left(\mathrm{e}^{i \theta}, t\right)^{2}} \mathrm{~d} \theta=\frac{-Q}{2 \pi a}=\frac{\dot{S}}{2 \pi a},
$$

where $a=f^{\prime}(0, t)$. In other words the area variation is controlled by the conformal radius of the domain $\Omega(t)$ with respect to the origin: $\dot{S} \leqslant 2 \pi a \dot{a}$. We point out here that generally $S \geqslant \pi a^{2}$ (this is a simple consequence of the area principle, see e.g. [10]). The equality in the above inequalities is attained for $\Omega(t)=\{z:|z|<a(t)\}$. In a similar manner S. D. Howison and Yu. E. Hohlov [14] estimated from below the distance from $\Gamma(t)$ to the origin.

The lower bound for $\dot{S}$ is unclear or at least much more difficult. One must estimate the integral mean

$$
\int_{0}^{2 \pi} \frac{1}{\left|f^{\prime}\left(\mathrm{e}^{i \theta}, t\right)\right|^{2}} \mathrm{~d} \theta
$$

from above. The cusp formation does not allow us to obtain a uniform (with respect to $t$ ) bound. But one can estimate (18) under some geometric conditions on the domain $\Omega(t)$ at time $t$. For example, this is possible if we assume that at time $t$ the domain $\Omega(t)$ is convex. Then the function $f$ is also convex, and thus $\frac{1}{2}$-starlike, say $\operatorname{Re} \zeta f^{\prime}(\zeta) / f(\zeta)>1 / 2$. Moreover the 1/4-Koebe theorem for convex functions yields $|f(\zeta)| \geqslant a / 2$. This implies the estimates

$$
\int_{0}^{2 \pi} \frac{1}{\left|f^{\prime}\left(\mathrm{e}^{i \theta}, t\right)\right|^{2}} \mathrm{~d} \theta<\frac{32 \pi}{a^{2}} \quad \text { and } \quad \dot{S}>\frac{\pi a}{8} \dot{a} .
$$

\section{Free boundary extends to infinity}

This model corresponds to the moving fluid front which for definiteness we suppose to be located to the right. More precisely, we denote by $\Omega(t)$ a simply connected domain in the phase $z$-plane occupied by the moving fluid and we suppose its moving boundary $\Gamma(t)=\partial \Omega(t)$ contains the point at infinity. With $z=x+i y$ one can construct a parameterization $\Gamma(t)$. Assuming a natural normalization for $\Gamma(t)$ close to $\infty$, we require that $\Gamma(t)$ is a vertical straight line near infinity. The initial situation is represented at time $t=0$ as $\Omega(0)=\Omega_{0}, \partial \Omega_{0}=\Gamma(0) \equiv \Gamma_{0}$. We construct the complex potential $W(z, t), \operatorname{Re} W=p$, where $p$ is, as usual, the pressure field in $\Omega(t)$. For each fixed $t$ the potential $W$ is an analytic function defined in $\Omega(t)$ which solves the problem

$$
\begin{aligned}
\nabla^{2} p & =0 & & \text { in } z \in \Omega(t), \\
p & =\gamma \kappa(z) & & \text { on } z \in \Gamma(t), \\
v_{n} & =-\frac{\partial p}{\partial \vec{n}} & & \text { on } z \in \Gamma(t) .
\end{aligned}
$$

We assume that the velocity tends to a constant value $Q$ as $x \rightarrow \infty$, which is positive when the fluid is removed to the right and negative otherwise. In terms of the potential $p$ we have $p(x, y, t) / x \rightarrow$ $-Q$ as $x \rightarrow \infty$ for any $t$ fixed. The existence problem has been discussed in the introduction. Note that for this case the local solvability and uniqueness were proved by M. Kimura [15].

We consider the auxiliary parametric complex $\zeta$-plane, $\zeta=\xi+i \eta$. The Riemann Mapping Theorem shows that there exists a conformal univalent map $f(\zeta, t)$ of the right half-plane $H^{+}=$ $\{\zeta: \operatorname{Re} \zeta>0\}$ into the phase plane, $f: H^{+} \rightarrow \Omega(t)$. The half-plane $H^{+}$is a natural param- 
etric domain for $\Omega$. The function $f(\zeta, 0)=f_{0}(\zeta)$ produces a parameterization of $\Gamma_{0}$. The smoothness of the boundary $\Gamma(t)$ and its behaviour in the neighbourhood of $\infty$ allow us to assume the normalization $f(\zeta, \cdot)=\zeta+a_{0}+a_{-1} / \zeta+\ldots, \zeta \sim \infty$, i.e. the function $f$ has an analytic continuation on the imaginary axis $\partial H^{+}$near $\infty$. Thus, the moving boundary is parameterized by $\Gamma(t)=f\left(\partial H^{+}, t\right)$. The normal exterior vector in 21 is given by the formula $\vec{n}=-f^{\prime}(\zeta, t) /\left|f^{\prime}(\zeta, t)\right|, \zeta \in \partial H^{+}$. We represent the curvature $\kappa$ of $\Gamma(t)$ in terms of $f$ as

$$
\kappa(i \eta, t)=\frac{\operatorname{Re} f^{\prime \prime}(i \eta, t) / f^{\prime}(i \eta, t)}{\left|f^{\prime}(i \eta, t)\right|}, \quad \eta \in(-\infty, \infty) .
$$

Repeating the calculation for the normal velocity as in Section 2 we come to the equation of Polubarinova-Galin type

$$
\operatorname{Re}\left(\dot{f}(\zeta, t) \overline{f^{\prime}(\zeta, t)}\right)=Q+\gamma H\left[\frac{i \operatorname{Im} S_{f}}{\left|f^{\prime}\right|}\right](\eta), \quad \operatorname{Re} \zeta=0,
$$

with the Hilbert transform defined as

$$
H[\psi](\eta):=\frac{1}{\pi i} \mathrm{P} \cdot \mathrm{V} \cdot \eta \int_{-\infty}^{\infty} \frac{\psi\left(i \eta^{\prime}\right)}{\eta^{\prime}-\eta} \mathrm{d} \eta^{\prime} .
$$

Note that a simple case $\gamma=0$ of the zero-surface-tension model has been considered in [13]. An equation similar to (22) for non-zero-surface-tension model has been obtained in [8].

Now we define a geometric property of $\Omega(t)$ which is natural for the problem in question. A simply connected domain $\Omega$ on the extended complex plane $\overline{\mathbb{C}}$ is said to be convex in the positive direction of the real axis if its complement can be covered by a family of non-intersecting parallel rays with the direction of $\mathbb{R}^{+}$. This definition can be found, e.g., in [10]. In the case of a smooth boundary this means that any point of the boundary is reachable from the right by a ray parallel to $\mathbb{R}^{+}$. A holomorphic univalent mapping $f(\zeta), \zeta \in H^{+}$, is said to be convex in the positive direction if $f\left(H^{+}\right)$is as above. A criterion for this property is provided by the following inequality:

$$
\operatorname{Re} f^{\prime}(\zeta)>0, \quad \zeta=\xi+i \eta \in H^{+} .
$$

If we consider $f$ in the closure of $H^{+}$, then the equality in 23 can be attained on the axis $\xi=0$. The level lines, i.e., the $f$-images of the lines $\xi=$ const, $\xi>0$, also bound domains convex in the positive direction as soon as the function $f$ is convex in the positive direction.

Denote by $T$ the blow-up time. The following theorem is proved by analogy with Theorem 1 .

THEOREM 2 Consider the fluid region expanding to the left $(Q<0)$. Let the surface tension $\gamma$ be sufficiently small. If the initial domain $\Omega_{0}$ is convex in the positive direction, then there exists $t=t(\gamma) \leqslant T$ such that for each $t \in[0, t(\gamma)]$ the domain $\Omega(t)$ is also convex in the positive direction.

Remark. In particular, in the zero-surface-tension model $t(0)=T$ and the above property is preserved for the whole time $t \in[0, T)$.

\section{REFERENCES}

1. Ceniceros, H. G., Hou, T. Y., \& Si, H. Numerical study of Hele-Shaw flow with suction. Phys. Fluids 11 (1999), 2471-2486. MR 2000d:76051 
2. Di Benedetto, E. \& Friedmann, A. The ill-posed Hele-Shaw model and the Stefan problem for supercooled water. Trans. Amer. Math. Soc. 282 (1984), 183-204. Zbl 0621.35102 MR 85g:35121

3. Duchon, J. \& Robert, R. Evolution d'une interface par capillarité et diffusion de volume, 1: existence locale en temps. Ann. Inst. H. Poincaré 1 (1984), 361-378. Zbl 0572.35051 MR 86i:35133

4. Elliott, C. M. \& Janovský, V., A variational inequality approach to Hele-Shaw flow with a moving boundary. Proc. Roy. Soc. Edinburgh Sect. A 88 (1981), 93-107. Zbl 0455.76043 MR 82d:76031

5. Escher, J. \& Simonett, G. On Hele-Shaw models with surface tension. Math. Res. Lett. 3 (1996), 467-474. Zbl 0860.35149 MR 97i:35145

6. Escher, J. \& Simonett, G. Classical solutions for Hele-Shaw models with surface tension. Adv. Differential Equations 2 (1997), 619-642. Zbl 0888.35142 MR 98b:35204

7. Escher, J. \& Simonett, G. A center manifold analysis for the Mullins-Sekerka model. J. Differential Equations 143 (1998), 267-292. Zbl 0896.35142 MR 98m:35228

8. Fokas, A. S. \& TAnveer, S. A Hele-Shaw problem and the second Painlevé transcendent. Math. Proc. Cambridge Philos. Soc. 124 (1998), 169-191. Zbl 0918.76020 MR 99b:7602

9. GALIN, L. A. Unsteady filtration with a free surface. Dokl. Akad. Nauk SSSR 47 (1945), 246-249 (in Russian). Zbl 0061.46202

10. Goodman, A. W. Univalent Functions. Vols. I, II. Mariner, 1983.

11. GuSTAFsSON, B. Applications of variational inequalities to a moving boundary problem for Hele-Shaw flows. SIAM J. Math. Anal. 16 (1985), 279-300. Zbl 0605.76043 MR 86m:35155

12. Hohlov, Yu. E., Prokhorov, D. V. \& VAsil'ev, A. Yu. On geometrical properties of free boundaries in the Hele-Shaw flows moving boundary problem. Lobachevskii J. Math. 1 (1998), 3-12 (electronic). Zbl 0939.76025 MR 99m:76054

13. Howison, S. D. Complex variable methods in Hele-Shaw moving boundary problems. European J. Appl. Math. 3 (1992), 209-224. Zbl 0759.76022

14. Howison, S. D. \& Hohlov, Yu. E. On the classification of solutions to the zero-surface-tension model for Hele-Shaw free boundary flows. Quart. Appl. Math. 54 (1994), 777-789. Zbl 0793.76093

15. Kimura, M. Time local existence of a moving boundary of the Hele-Shaw flow with suction. European J. Appl. Math. 10 (1999), 581-605. Zbl 0955.76020 MR 2001e:76038

16. MCLean, J. W. \& Saffman, P. G. The effect of surface tension on the shape of fingers in a Hele Shaw cell. J. Fluid Mech. 102 (1981), 455-469. Zbl 0472.76111

17. MuskhelishviLi, N. I. Singular Integral Equations. Noordhoff, Groningen, 1953. Zbl 0051.33203

18. Ockendon, H. \& Ockendon, J. R. Viscous Flow. Cambridge Univ. Press, 1995. Zbl 0837.76001 MR 95k:76025

19. Polubarinova-Kochina, P. Ya. On a problem of the motion of the contour of a petroleum shell. Dokl. Akad. Nauk SSSR 47 (1945), 254-257 (in Russian).

20. Polubarinova-Kochina, P. Ya. Concerning unsteady motions in the theory of filtration. Prikl. Mat. Mekh. 9 (1945), 79-90 (in Russian).

21. Pommerenke, C. Univalent Functions (with a chapter on quadratic differentials by G. Jensen). Vandenhoeck \& Ruprecht, Göttingen, 1975. Zbl 0298.30014

22. Pommerenke, C. Boundary Behaviour of Conformal Maps. Springer, Berlin, 1992. Zbl 0762.30001

23. Prokert, G. Existence results for Hele-Shaw flow driven by surface tension. European J. Appl. Math. 9 (1998), 195-221. Zbl 0919.35005 MR 2000d:76052

24. QIng, N. \& Tian, F. R. Singularities in Hele-Shaw flows. SIAM J. Appl. Math. 58 (1998), 34-54. Zbl 0917.76020 MR 2000c:76023

25. REISSIG, M. \& VON WOLFERSDORF, L. A simplified proof for a moving boundary problem for HeleShaw flows in the plane. Ark. Mat. 31 (1993), 101-116. Zbl 0802.35168 
26. Primicerio, M. \& Rodrigues, J.-F. The Hele-Shaw problem with nonlocal injection condition. Nonlinear Mathematical Problems in Industry, II (Iwaki, 1992), GAKUTO Internat. Ser. Math. Sci. Appl. 2, Gakkōtosho, Tokyo, 1993, 375-390. Zbl 0875.35157 MR 96j:3527

27. Saffman, P. G. \& TAYlor, G. I. The penetration of a fluid into a porous medium or Hele-Shaw cell containing a more viscous liquid. Proc. Roy. Soc. London Ser. A 245 (1958), 312-329. Zbl 0086.41603

28. Siegel, M., TANVEER, S., \& DAI, W. S. Singular effects of surface tension in evolving Hele-Shaw flows. J. Fluid Mech. 323 (1996), 201-236. Zbl0885.76022 MR 97h:76041

29. Tanveer, S. Evolution of Hele-Shaw interface for small surface tension. Philos. Trans. Roy. Soc. London Ser. A 343 (1993), 155-204. Zbl 0778.76029

30. TIAN, F.-R. Hele-Shaw problems in multidimensional spaces. J. Nonlinear Sci. 10 (2000), 275-290. Zbl 0955.76024 MR 2000m:76041

31. Vinogradov, Yu. P. \& Kufarev, P. P. On a problem of filtration. Prikl. Mat. Mekh. 12 (1948), 181-198 (in Russian). Zbl 0032.27901 MR 9,540j 